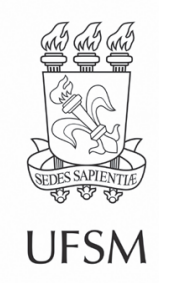

\title{
Artigos
}

\section{Distribuição diamétrica de três espécies de Lecythidaceae após exploração de impacto reduzido na Amazônia Oriental}

\author{
Diametric distribution of three species of Lecythidaceae after reduced \\ impact logging in Eastern Amazon
}

\author{
Loarena Leal Cruz ${ }^{1}$, Nelson Yoshihiro Nakajima $" \odot$, \\ Renildo Medeiros da Silva "II๑, Roberto Tuyoshi Hosokawa " $\odot$,

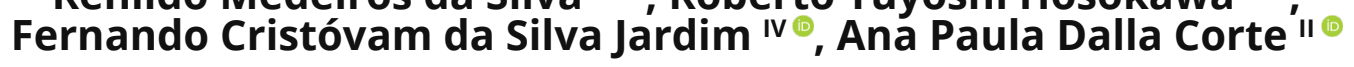 \\ ' Universidade Federal de Lavras, Lavras, MG, Brasil \\ " Universidade Federal do Paraná, Curitiba, PR, Brasil \\ III Pesquisador Autônomo, Abel Figueiredo,PA, Brasil \\ iv Universidade Federal Rural da Amazônia, Belém, PA, Brasil
}

\section{RESUMO}

Eschweilera coriacea, Eschweilera ovata e Lecythis idatimon são espécies arbóreas tropicais potencialmente comerciais, portanto, amplamente colhidas na Amazônia Oriental. O objetivo deste trabalho foi avaliar o desempenho de três funções de densidade probabilísticas no ajuste da distribuição diamétrica de três espécies de Lecythidaceae, para o período entre um e dez anos após a colheita florestal, em área manejada, em Moju, Pará. Foram selecionadas nove clareiras provenientes da exploração de impacto reduzido realizada em 1997. Em torno de cada clareira foram instaladas quatro faixas de $10 \times 50 \mathrm{~m}$, começando da bordadura da clareira para dentro da floresta, nas direções norte, sul, leste e oeste, perfazendo uma amostragem de 1,8 ha. Foram mensurados todos os indivíduos com DAP $\geq 5 \mathrm{~cm}$ em duas medições (1998 e 2007). Foram testadas as funções de densidade probabilísticas Weibull 3P, Gamma e Weber. Para a seleção do melhor modelo, foram avaliados o valor D de Kolmogorov - Smirnov $(a=0,05)$, erro padrão da estimativa (Syx\%), coeficiente de determinação ajustado ( $R^{2}$ ajustado) e análise gráfica das curvas de frequências estimadas sobre o histograma das observadas. Em ambos os anos, a função Weibull 3P apresentou as melhores estatísticas de ajuste e maior precisão para descrever a distribuição diamétrica de Eschweilera coriacea e Lecythis idatimon, e o modelo de Weber para Eschweilera ovata. A função Gamma foi descartada para todas as espécies. Os resultados indicam a não viabilidade do uso de uma mesma função para descrever diferentes espécies, necessitando considerar as características intrínsecas de cada espécie.

Palavras-chave: Exploração florestal; Diâmetro arbóreo; Função de densidade probabilística 


\section{ABSTRACT}

Eschweilera coriacea, Eschweilera ovata and Lecythis idatimon are potentially commercial tropical species, therefore, widely harvested in Eastern Amazon. This research aims to evaluate the performance of three probabilistic density functions in the adjustment of the diametric distribution of three Lecythidaceae species, after one and ten years of the forest harvest, in a managed area, in the municipality of Moju, Pará state. Nine gaps proceeding from the reduced impact logging carried out in 1997 were studied. Around each gap, four $10 \times 50 \mathrm{~m}$ strips were installed, starting from the edge of the gap into the forest, in the northern, southern, eastern and western directions, in a sampling of 1,8 ha. All individuals with $\mathrm{DBH} \geq 5 \mathrm{~cm}$ were measured in two measurements (1998 and 2007). Probabilistic density functions Weibull 3P, Gamma and Weber were tested. For selecting the best model, we analyze the Kolmogorov Smirnov D value $(a=0.05)$, standard error of the estimate (Syx\%), the adjusted determination coefficient (adjusted $\mathrm{R}^{2}$ ) and the graphical analysis of the frequency curves estimated on the histogram of the observed ones. In both years, the Weibull 3P function presented the best fit and accuracy statistics to describe the diametric distribution of Eschweilera coriacea and Lecythis idatimon, and the Weber model for Eschweilera ovata. Gamma function was discarded for all species. The results indicate the non-feasibility of using the same function to describe different species, needing to consider the intrinsic characteristics to each species.

Keywords: Forest exploration; Tree diameter; Probabilistic density function

\section{INTRODUÇÃO}

A Amazônia brasileira representa cerca de um terço das florestas tropicais do mundo, caracterizando-se por sua elevada riqueza de espécies, das quais se estima que mais de 350 sejam extraídas e comercializadas no setor madeireiro (VERÍSSIMO; PEREIRA, 2014). Dentre essas, destacam-se as pertencentes à Lecythidaceae, que concentra sua distribuição na região neotropical, constituída por cerca de 25 gêneros e 300 espécies (MORI, 2001; MORI; PRANCE, 1990). No Brasil, a família apresenta 10 gêneros e em torno de 120 espécies, com aproximadamente 45\% delas endêmicas do país, sendo o Amazonas o principal centro de diversidade da família (SMITH et al., 2012).

Lecythidaceae é uma das famílias botânicas ecologicamente mais dominantes da flora arbórea amazônica (TER STEEGE et al., 2013). Ter Steege et al. (2006), em um estudo ecológico em grande escala, constataram que é a terceira mais abundante de toda a Amazônia, superada somente por Fabaceae e Sapotaceae. Os gêneros de 
Lecythidaceae mais representativos em número de espécies são Eschweilera e Lecythis (SMITH et al., 2016). Suas espécies possuem grande importância socioeconômica, devido ao alto potencial madeireiro, destacando-se: Eschweilera coriacea (DC.) S. A. Mori (matamatá branco); Eschweilera ovata (Cambess.) Mart. ex Miers (matamatá preto); e Lecythis idatimon Aubl. (ripeiro), espécies amplamente colhidas para produção de madeira no Pará (SEMAS, 2018).

Entretanto, as informações acerca da autoecologia e dinâmica das espécies comerciais da Amazônia brasileira são ainda incipientes. É necessário conhecer as características de cada espécie para que práticas silviculturais e de conservação adequadas ao manejo sejam adotadas. Estudos sobre o comportamento dessas espécies, principalmente em relação ao diâmetro, que é bastante influenciado pela colheita de madeira, são de suma importância para dar suporte à sustentabilidade das atividades florestais, em especial, no intuito de definir corretamente seu ciclo de corte.

A distribuição diamétrica permite identificar diferentes tipologias florestais. Quando aliada às sucessivas medições, torna-se uma ferramenta poderosa na avaliação da dinâmica de florestas nativas e plantadas (MACHADO et al., 2009). Atualmente, a melhor forma de descrever a estrutura diamétrica de uma floresta ou espécie é através do emprego de funções de densidade probabilísticas (FDP), pois permitem obter a probabilidade das árvores ocorrerem dentro de determinados intervalos de diâmetro.

Devido às características intrínsecas à cada espécie, necessita-se que os modelos utilizados sejam devidamente testados e selecionados, identificando o que proporciona maior consistência para descrever o comportamento da variável de interesse (MACHADO et al., 2010). São várias as distribuições probabilísticas que têm sido usadas para descrever as distribuições diamétricas das espécies florestais nativas, como Normal, Log-normal, Gamma, Beta, SB de Johnson, Weibull (2 e 3 parâmetros) e Weber, essa última desenvolvida especificamente para a área florestal (DALLA LANA et al., 2013).

Dessa forma, buscou-se avaliar a distribuição diamétrica de três espécies de 
Lecythidaceae: Eschweilera coriacea, Eschweilera ovata e Lecythis idatimon, por meio do ajuste de três funções de densidade probabilísticas em uma floresta manejada após um e dez anos ocorrida a Exploração de Impacto Reduzido no município de Moju, Pará.

\section{MATERIAL E MÉTODOS}

\section{1 Área de estudo}

O estudo foi conduzido em uma área de 200 ha, pertencente ao Campo Experimental da Embrapa Amazônia Oriental, Km 30 da Rodovia PA-150, no município de Moju, Pará, sob as coordenadas $2^{\circ} 07^{\prime} 30^{\prime \prime}$ a $2^{\circ} 12^{\prime} 06^{\prime \prime}$ S e $48^{\circ} 46^{\prime} 57^{\prime \prime}$ a $48^{\circ} 48^{\prime} 30^{\prime \prime}$ W (Figura 1). O clima da região é do tipo $\mathrm{Am}_{\mathrm{i}}$ (quente e úmido), segundo a classificação de Köppen, com temperatura média anual de $26^{\circ} \mathrm{C}$. O período seco ocorre de agosto a outubro e o período mais chuvoso de fevereiro a abril. A precipitação pluviométrica anual é de 2.000 a 3.000 mm, com umidade relativa do ar em torno de $85 \%$. O relevo é plano, com declives de até $3 \%$. Os solos predominantes são classificados como Latossolos Amarelos e Argissolos Amarelos típicos, com suas fases pedregosas (COSTA et al., 1998).

A tipologia da vegetação da área experimental é Floresta Ombrófila Densa ou Floresta Tropical de Terra Firme (IBGE, 2012). A altura do dossel varia de 25 a 35 metros, sendo a área caracterizada pela presença de algumas palmeiras no subbosque, predominando as famílias Lecythidaceae, Violaceae, Sapotaceae, Burseraceae, Moraceae e Fabaceae; e as espécies Rinorea guianensis Aubl. (acariquarana), Eschweilera coriacea (DC.) S. A. Mori (matamatá branco), Lecythis idatimon Aubl. (ripeiro), Protium pilosum (Cuatrec.) Daly (breu) e Vouacapoua americana Aubl. (acapu) (COSTA et al., 1998; JARDIM, VASCONCELOS, 2006).

A área estudada sofreu exploração florestal seletiva de impacto reduzido, de acordo com a legislação vigente em 1997, de onde foi retirada um total de 3,3 árvores 
ha-1e um volume médio de $23 \mathrm{~m}^{3} \mathrm{ha}^{-1}$ (REIS, 2012). Foram exploradas 24 espécies, destacando-se Piptadenia suaveolens Miq. (Timborana), Manilkara elata (Allemão ex Miq.) Monach. (Maçaranduba) e Vouacapoua americana Aubl. (Acapú), por possuírem os maiores volumes. Com o intuito de verificar o comportamento das espécies sob influência do manejo florestal, foram selecionadas aleatoriamente 09 clareiras provenientes/oriundas da derrubada de 39 árvores, com áreas que variaram de 231 a $748 \mathrm{~m}^{2}$, considerando clareira como toda área da floresta com dossel descontínuo, estimadas através da média de dois diâmetros, o maior da clareira e seu diâmetro perpendicular, sendo determinado o centro de cada clareira por procedimentos topográficos.

Figura 1 - Localização do Campo Experimental da Embrapa Amazônia Oriental, no município de Moju - PA

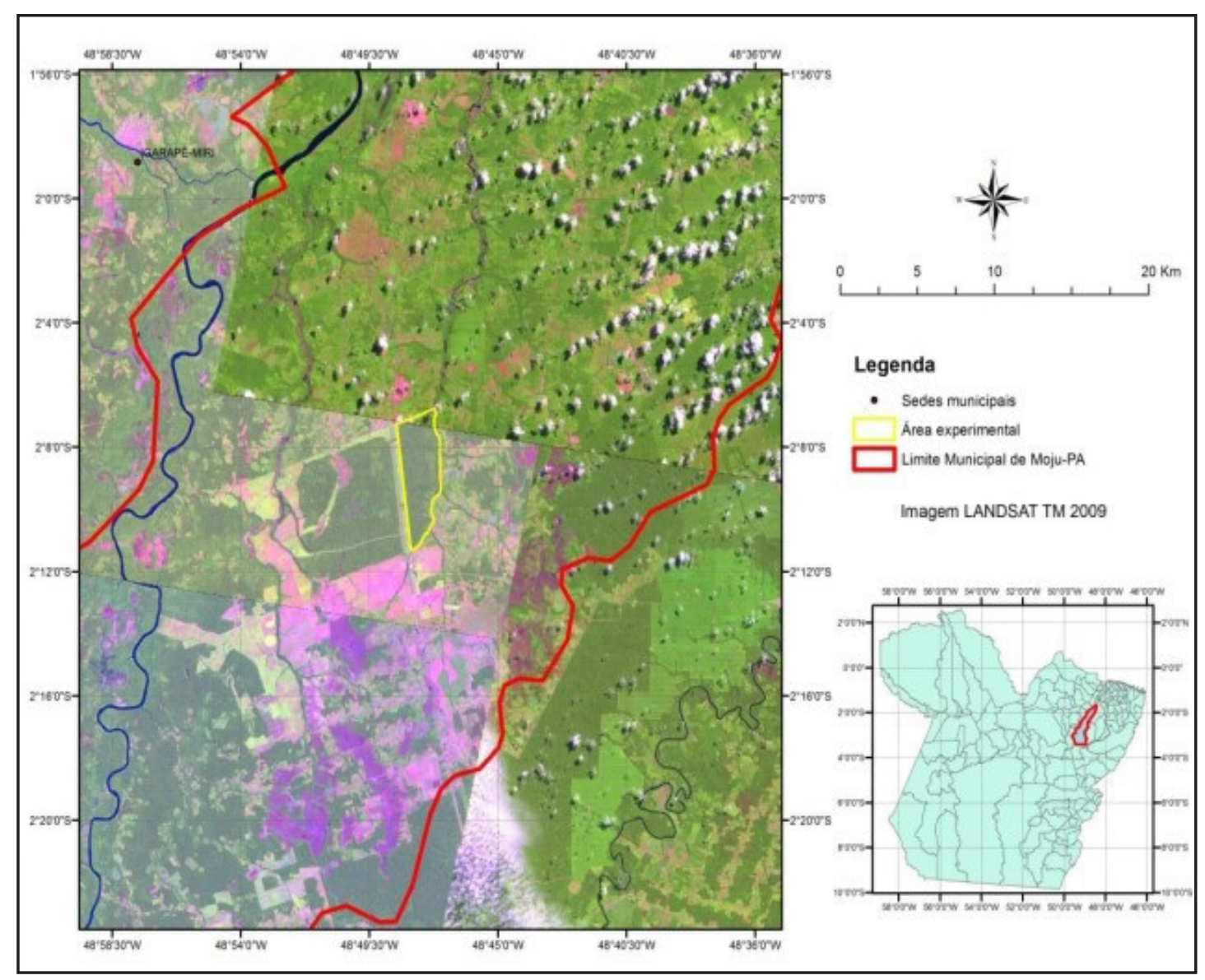

Fonte: Reis (2012) 
No entorno de cada clareira, foram instaladas faixas permanentes de $10 \times 50 \mathrm{~m}$ $\left(500 \mathrm{~m}^{2}\right)$, começando na bordadura da clareira para dentro da floresta, nas direções Norte, Sul, Leste e Oeste, portanto, totalizando quatro faixas por clareira (Figura 2), perfazendo uma amostragem de 1,8 ha. Nestas, foram registrados, medidos, identificados e plaqueteados todos os indivíduos presentes com DAP $\geq 5 \mathrm{~cm}$. Foram realizadas duas medições nos anos de 1998 (um ano após a colheita de madeira) e 2007 (dez anos após a colheita).

Figura 2 - Distribuição das faixas em relação às clareiras de colheita florestal, no Campo Experimental da Embrapa Amazônia Oriental, no município de Moju - PA

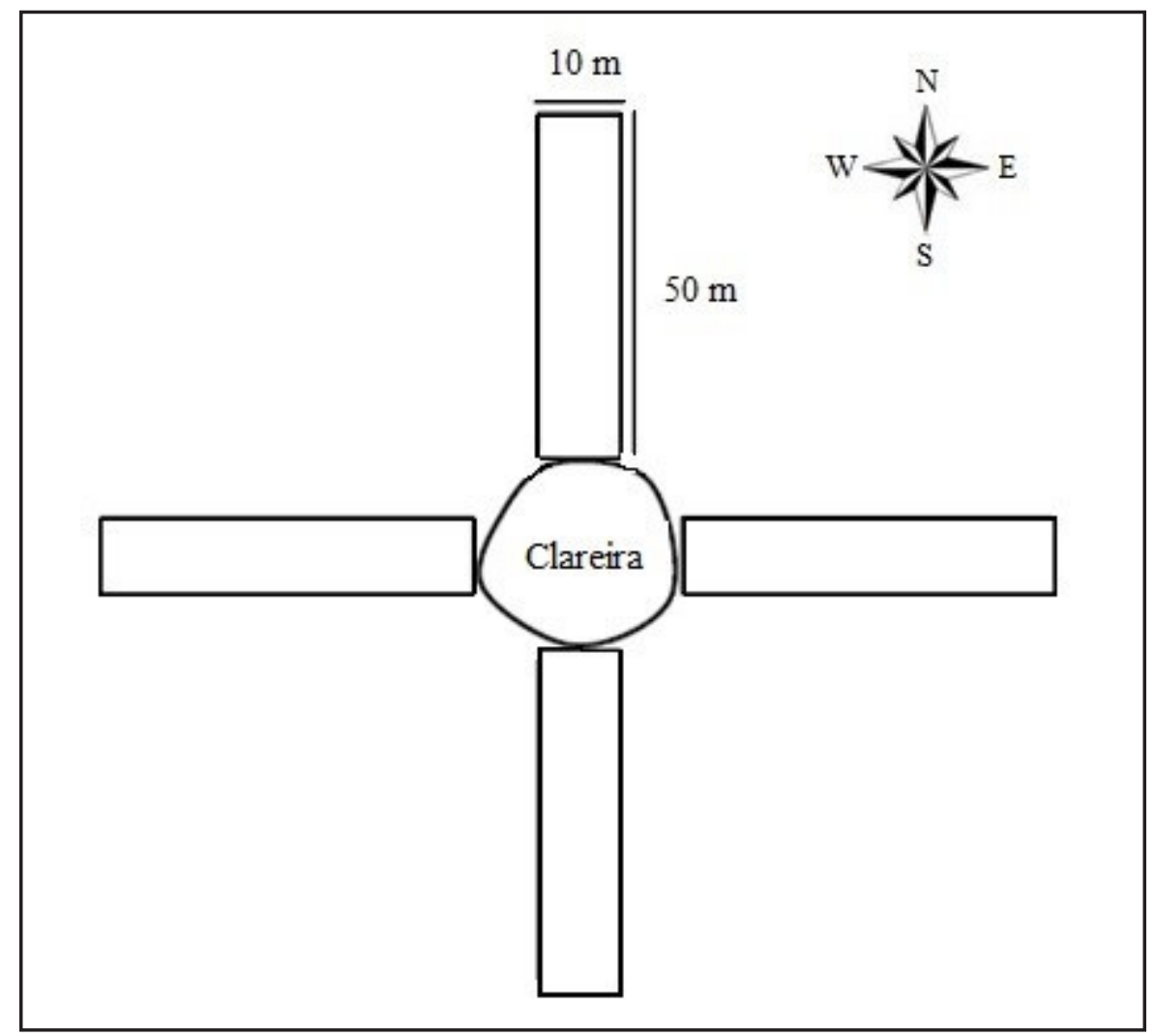

Fonte: Autores (2020)

\subsection{Análise dos dados}

As funções de densidade de probabilidade testadas para obter a frequência de distribuição da variável diâmetro foram “Weibull 3 parâmetros” ("Weibull 3P”), “Gamma” 
e "Weber" (Tabela 1). Para o ajuste das distribuições probabilísticas foram selecionadas três espécies da família Lecythidaceae, em razão de sua representatividade na área do presente estudo e potencial econômico, selecionadas a partir do Índice de Valor de Importância (IVI) (ARAÚJO, 2011), as quais são: Eschweilera coriaceae (DC.) S. A. Mori, Eschweilera ovata (Cambess.) Mart. ex Miers e Lecythis idatimon Aubl. Para cada uma das espécies estudadas, foram ajustadas as três funções de densidade de probabilidade para cada ano analisado (1998 e 2007).

Tabela 1 - Funções de densidade probabilísticas testadas

\begin{tabular}{lcc}
\hline Função & Funções de densidade probabilísticas (FDP) & $\begin{array}{c}\text { Fatores } \\
\text { condicionantes }\end{array}$ \\
\hline Weibull 3P & $f(x)=\frac{c}{b} \cdot \frac{x-a^{c-1}}{b} \cdot e^{-\frac{x-a^{c}}{b}}$ & $\begin{array}{c}a \leq x<\infty \\
a \geq 0 \\
b, c>0\end{array}$ \\
\hline Gamma & $f(x)=\frac{x^{\alpha-1} e^{-x / \beta}}{\beta^{\alpha} \Gamma(\alpha)}$ & $\begin{array}{c}x \geq x_{\min } \\
\alpha, \beta>0\end{array}$ \\
& & $-\infty<x_{\min }<+\infty$ \\
Weber & $x^{a}$ & $\begin{array}{c}0 \leq x< \pm \infty \\
d \geq a+1 \\
d \neq a+2 \\
d \neq a+3 \\
a, b, c>0\end{array}$ \\
\hline
\end{tabular}

Fonte: Autores (2020)

Em que: $f(x)=$ Função densidade de probabilidade da variável $x ; x=$ Variável aleatória; $x_{\min }=$ Valor mínimo de $x ; \pi=$ Constante PI $(3,1416 \ldots) ; e=$ Constante de Euler $(2,7182 \ldots) ; a, b$, $c, d, \alpha, \beta, \delta, \varepsilon, \gamma, \lambda=$ Parâmetros a serem estimados.

Na modelagem das distribuições de frequência diamétrica, adotou-se um intervalo de classe de cinco centímetros para as espécies. O ajuste das funções ocorreu por regressão linear, pelo método dos mínimos quadrados com múltiplas interações, segundo procedimento descrito por Marquardt (1963), utilizando os softwares "Table Curve 2D" (AISN SOFTWARE, 1994) e MS EXCEL 2010. Após os ajustes, foram traçadas curvas de frequências estimadas sobre o histograma de frequências observadas para cada classe de diâmetro. A comparação e escolha da função que melhor descreveu a distribuição diamétrica das espécies estudadas consideraram a análise gráfica dos 
valores estimados e observados em cada função probabilística nos diferentes anos, o erro padrão de estimativa em porcentagem (Syx\%), que indica quão próximos os valores estimados estão dos valores observados, quanto mais próximo de zero mais preciso será o modelo, o coeficiente de determinação ajustado ( $R^{2}$ ajustado), o qual identifica o quanto a variação total é explicada pelo modelo, quanto maior o valor encontrado, melhor será o ajuste e, pelo teste estatístico de Kolmogorov-Smirnov (KS), a um nível de $5 \%$ de probabilidade $(a=0,05)$, cuja hipótese nula $\left(H_{0}\right)$ consiste na aderência entre dados observados e ajustados; e a hipótese alternativa $\left(H_{1}\right)$ pressupõe a não aderência dos mesmos. Esse teste compara a máxima diferença entre as frequências acumuladas observada e estimada, em razão do número de observações, sendo o ponto de maior divergência entre as duas distribuições chamado de valor $D$ de Kolmogorov-Smirnov (SCOLFORO, 2006). Assim, se D calculado < D tabelado ( $\alpha=5 \%$ ), aceita-se a hipótese de que os diâmetros observados seguem as distribuições testadas $\left(H_{0}\right)$, caso seja verificado o contrário ( $D$ calculado > D tabelado e, consequentemente, valores significativos a $5 \%$ de probabilidade no teste bilateral), rejeita-se a hipótese e os diâmetros observados não seguem as distribuições testadas $\left(H_{1}\right)$. A função que apresentar o menor D calculado indicará a melhor aderência entre as distribuições diamétricas (SCOLFORO, 2006).

A seleção da melhor função de densidade de probabilidade deu-se primeiramente com a verificação da aderência e posteriormente com a análise da função que apresentou menor erro padrão da estimativa em porcentagem (Syx\%) e melhor coeficiente de determinação ajustado ( $R^{2}$ ajustado). Por último realizouse a análise gráfica das funções, comparando a distribuição original com as funções densidade de probabilidade.

\section{RESULTADOS E DISCUSSÃO}

Nos anos de 1998 e 2007, respectivamente, foram verificados 107 e 109 indha-1 de Eschweilera coriacea, 46 e 39 indha $^{-1}$ de Eschweilera ovata, 118 e 135 indha $^{-1}$ de Lecythis 
idatimon. Em média, considerando os dois anos de medição, cerca de 33\%, 30\% e 44\% dos indivíduos amostrados de Eschweilera coriacea, Eschweilera ovata e Lecythis idatimon, respectivamente, apresentaram diâmetro abaixo de $10 \mathrm{~cm}$, sendo verificado redução progressiva da frequência mediante o aumento da classe de inclusão de diâmetro.

Os parâmetros obtidos no ajuste dos modelos de distribuição de densidade probabilística para obtenção da frequência estimada de árvores por classe diamétrica das três espécies estudadas, para um e dez anos após a colheita florestal, são apresentados na Tabela 2.

Tabela 2 - Parâmetros de ajuste estimados das distribuições Weibull 3P, Gamma e Weber, testadas para as populações de Lecythidaceae estudadas, nos anos avaliados, em uma floresta de terra firme, explorada sob impacto reduzido, em Moju - PA

\begin{tabular}{|c|c|c|c|c|c|c|c|}
\hline \multirow{2}{*}{ Função } & \multirow{2}{*}{ Parâmetros } & \multicolumn{2}{|c|}{ Eschweilera coriacea } & \multicolumn{2}{|c|}{ Eschweilera ovata } & \multicolumn{2}{|c|}{ Lecythis idatimon } \\
\hline & & 1998 & 2007 & 1998 & 2007 & 1998 & 2007 \\
\hline \multirow{3}{*}{$\begin{array}{l}\text { Weibull } \\
3 P\end{array}$} & a & 5,9739 & 6,2841 & 7,5000 & 7,5000 & 6,0964 & 7,4129 \\
\hline & $b$ & 16,5354 & 17,3955 & 21,3297 & 20,4778 & 12,8762 & 29,5031 \\
\hline & c & 0,8730 & 0,8262 & 0,9928 & 0,9780 & 0,8728 & 0,7752 \\
\hline \multirow{2}{*}{ Gamma } & $a$ & 1,8586 & 1,8540 & 1,9216 & 2,1204 & 2,4510 & 3,0292 \\
\hline & $\beta$ & 7,1287 & 6,6401 & 8,9618 & 6,6247 & 4,5542 & 2,6644 \\
\hline \multirow{4}{*}{ Weber } & a & $-0,5540$ & $-0,8183$ & $-0,2368$ & $-0,5475$ & $-0,6746$ & $-1,0064$ \\
\hline & $b$ & 1,0245 & 1,0105 & 1,0145 & 1,0255 & 1,0095 & $-74987,7772$ \\
\hline & c & 0,0007 & 0,0003 & 0,0002 & 0,0006 & 0,0005 & 10003,2411 \\
\hline & $d$ & 55,5816 & 81,5717 & 155,2436 & 56,0140 & 90,4323 & 0,0869 \\
\hline
\end{tabular}

Fonte: Autores (2020)

De maneira geral, para todas as espécies estudadas, os parâmetros obtidos a partir das funções Weibull 3P e Gamma mostraram-se similares para os dois períodos analisados, com exceção dos parâmetros de locação "b" para Weibull 3P e " $\beta$ " para Gamma para Lecythis idatimon. Já para a função de Weber, não houve tendência de similaridade entre as estimativas avaliadas, mostrando uma grande dispersão de valores, principalmente para Lecythis idatimon no ano de 2007. Machado et al. (2010) afirmam que a ausência dessa tendência demonstra a não viabilidade do uso de uma 
mesma função para avaliar diferentes espécies, necessitando identificar qual função que melhor se adequa às características de cada espécie.

Os valores da estatística das funções de densidade de probabilidade estudadas são apresentados na Tabela 3. A hipótese nula de que ocorre aderência entre dados observados e ajustados foi confirmada apenas para as funções Weibull 3P e Weber, uma vez que foram observados valores de $D$ calculado menores que $D$ tabelado ( $a$ $=0,05)$ para todas as espécies estudadas, nos diferentes anos avaliados. Em termos estatísticos, isso implica que as frequências estimadas e observadas foram similares a partir do ajuste dessas funções. Em contrapartida, a função Gamma foi considerada inadequada para descrever a distribuição diamétrica de Eschweilera coriacea e Lecythis idatimon em ambos os anos, considerando que a significância apresentada indica o aceite da hipótese da não aderência entre dados ajustados e observados.

Tabela 3 - Valores de D tabelado (D Tab.), e D calculado (D Cal.), obtidos por meio do teste estatístico de K-S, para as populações de Lecythidaceae estudadas, nos anos avaliados, em uma floresta de terra firme, em Moju - PA

\begin{tabular}{|c|c|c|c|c|c|c|c|}
\hline \multirow{2}{*}{ Função } & \multirow{2}{*}{ K-S } & \multicolumn{2}{|c|}{ Eschweilera coriacea } & \multicolumn{2}{|c|}{ Eschweilera ovata } & \multicolumn{2}{|c|}{ Lecythis idatimon } \\
\hline & & 1998 & 2007 & 1998 & 2007 & 1998 & 2007 \\
\hline \multirow{4}{*}{$\begin{array}{l}\text { Weibull } \\
3 \mathrm{P}\end{array}$} & D Tab. (5\%) & 0,1315 & 0,1303 & 0,2005 & 0,2178 & 0,1252 & 0,1171 \\
\hline & D Cal. & 0,0199 & 0,0238 & 0,0300 & 0,1613 & 0,0291 & 0,0371 \\
\hline & $\mathrm{R}^{2}$ ajustado & 0,9684 & 0,9614 & 0,9592 & 0,9592 & 0,9718 & 0,9570 \\
\hline & Syx (\%) & 16,5103 & 1,9631 & 0,5930 & 0,5930 & 2,2301 & 3,7968 \\
\hline \multirow{4}{*}{ Gamma } & D Tab. (5\%) & 0,1315 & 0,1303 & 0,2005 & 0,2178 & 0,1252 & 0,1171 \\
\hline & D Cal. & $0,1883^{*}$ & $0,2097^{*}$ & 0,1548 & 0,1658 & $0,1855^{*}$ & $0,2826^{*}$ \\
\hline & $\mathrm{R}^{2}$ ajustado & 0,8965 & 0,8657 & 0,7572 & 0,5510 & 0,8601 & 0,7648 \\
\hline & Syx (\%) & 3,0082 & 3,6839 & 1,4771 & 2,4675 & 5,0742 & 9,0656 \\
\hline \multirow{4}{*}{ Weber } & D Tab. (5\%) & 0,1315 & 0,1303 & 0,2005 & 0,2178 & 0,1252 & 0,0567 \\
\hline & D Cal. & 0,0310 & 0,0291 & 0,0167 & 0,0444 & 0,0327 & 0,1171 \\
\hline & $\mathrm{R}^{2}$ ajustado & 0,9612 & 0,9505 & 0,9426 & 0,4649 & 0,9596 & 0,9359 \\
\hline & Syx (\%) & 1,8160 & 2,2058 & 0,6813 & 2,4592 & 2,5850 & 4,4897 \\
\hline
\end{tabular}

Fonte: Autores (2020)

Em que: *Significativo a $5 \%$ de probabilidade no teste bilateral; $\mathrm{R}^{2}$ ajustado = coeficiente de determinação ajustado; Syx (\%) = erro padrão da estimativa em porcentagem. 
Dentre as funções de densidade probabilísticas testadas, em ambos os anos, Weibull 3P demonstrou maior eficiência para representar as populações de Eschweilera coriacea e Lecythis idatimon. Segundo Nascimento et al. (2012), em especial, essa função é preferencialmente empregada nas ciências florestais devido suas características de maior flexibilidade, precisão e correlação entre seus coeficientes e atributos populacionais da floresta. Apesar de todas as funções haverem sido satisfatórias para descrever a distribuição diamétrica de Eschweilera ovata, a função que melhor a representou nas duas ocasiões foi a de Weber.

Foram traçadas as curvas de frequências estimadas sobre o histograma das frequências observadas para as três espécies de Lecythidaceae estudadas nas Figuras 3, 4 e 5, nos anos avaliados.

Um ano após a colheita seletiva de madeira, as curvas de distribuição diamétrica se apresentaram na forma exponencial negativa ("J invertido"), isto é, com grande concentração de indivíduos nos centros de classe de diâmetro inferiores, para todas as populações estudadas. Esses resultados corroboram com os obtidos por Dalla Lana et al. (2013), que estudando uma população de Eschweilera ovata, verificaram o mesmo padrão para a espécie, em um fragmento de Floresta Atlântica com vegetação do tipo Floresta Ombrófila Densa em Igarassu, Pernambuco. Estudos mostram que esse padrão de distribuição diamétrica é comum em florestas tropicais (SANTOS et al., 2013; TÉO et al., 2015; ORELLANA et al., 2014). Essa distribuição é característica das espécies pertencentes às florestas inequiâneas (ASSMANN, 1970) e que apresentam potencial de recuperação devido à representatividade da regeneração natural, através de estoques de populações jovens (MACHADO et al., 2009; HARPER, 1990), em especial, após um ano de ocorrida a exploração florestal as espécies estudadas indicaram que a floresta manteve suas características naturais.

No entanto, após transcorridos dez anos da intervenção na área, observou-se uma distribuição diamétrica irregular. Excetuando-se Eschweilera coriacea, que manteve sua distribuição diamétrica similar nos dois períodos analisados, para as demais 
espécies ocorreu uma queda abrupta das curvas de frequências, principalmente na segunda classe diamétrica. Esse fato concorda com Lima et al. (2002), que ao estudarem três áreas de exploração madeireira na Amazônia, caracterizaram Eschweilera coriacea como uma espécie de crescimento lento. Entretanto, sua densidade após a exploração não diminuiu, o que indica que essa espécie é pioneira, conseguindo sobreviver em clareiras e ao redor delas, que, provavelmente, estará disponível para corte no próximo ciclo, ratificando assim o seu potencial.

Figura 3 - Curvas de distribuição diamétrica de Eschweilera coriacea estimadas pelas três funções de densidade de probabilidade, sobre o histograma de frequências observadas, um e dez anos após a exploração florestal, na Amazônia oriental, Moju - PA

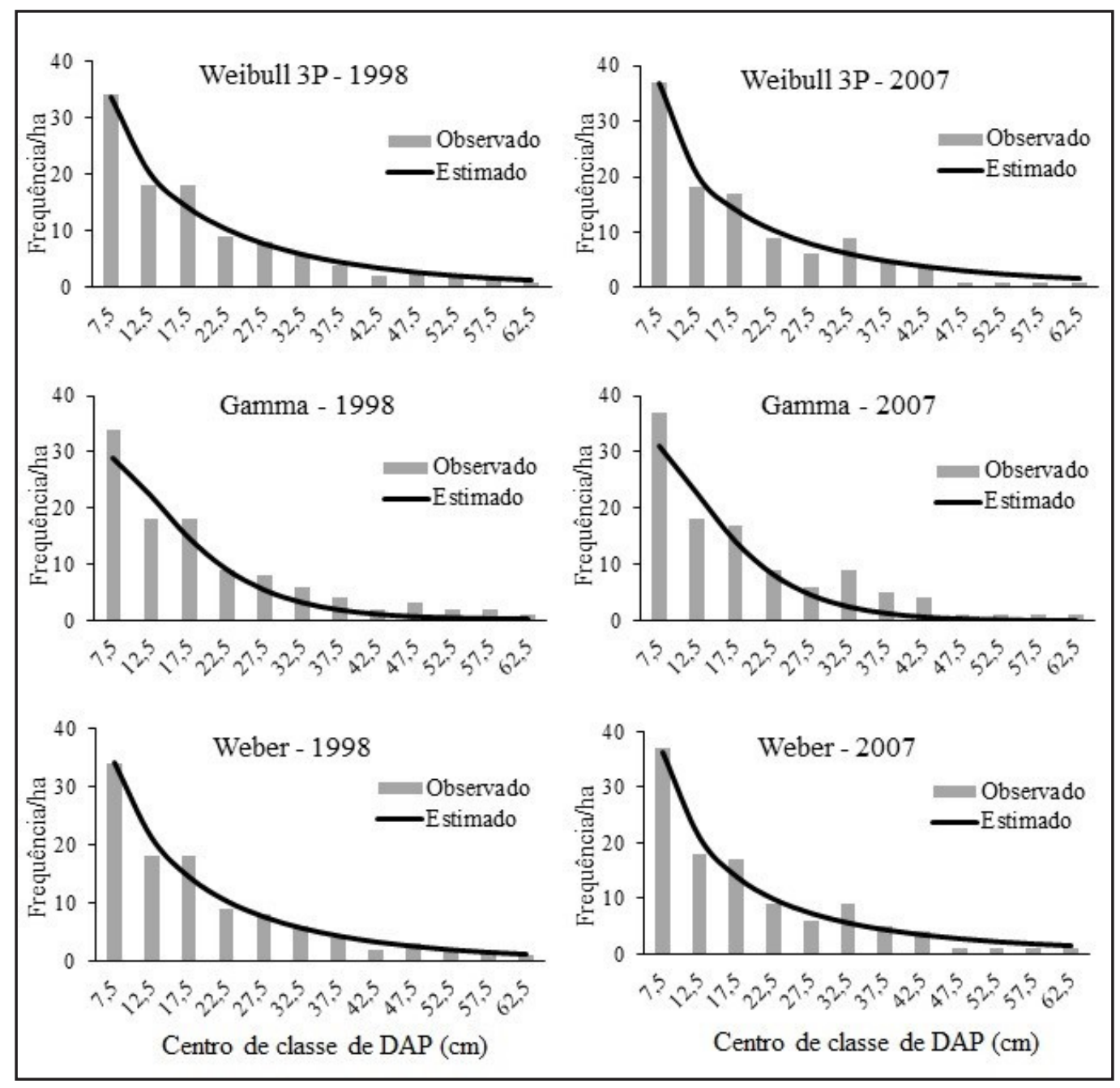

Fonte: Autores (2020) 
Orellana et al. (2014), ao estudarem um fragmento de floresta ombrófila mista afirmaram que a forma das distribuições diamétricas das espécies são influenciadas pelas suas características ecológicas, e dependendo do regime de manejo em que uma espécie é submetida poderá descaracterizar sua estrutura diamétrica e, consequentemente, sua distribuição.

Figura 4 - Curvas de distribuição diamétrica de Eschweilera ovata estimadas pelas três funções de densidade de probabilidade, sobre o histograma de frequências observadas, um e dez anos após a exploração florestal, na Amazônia oriental, Moju-PA

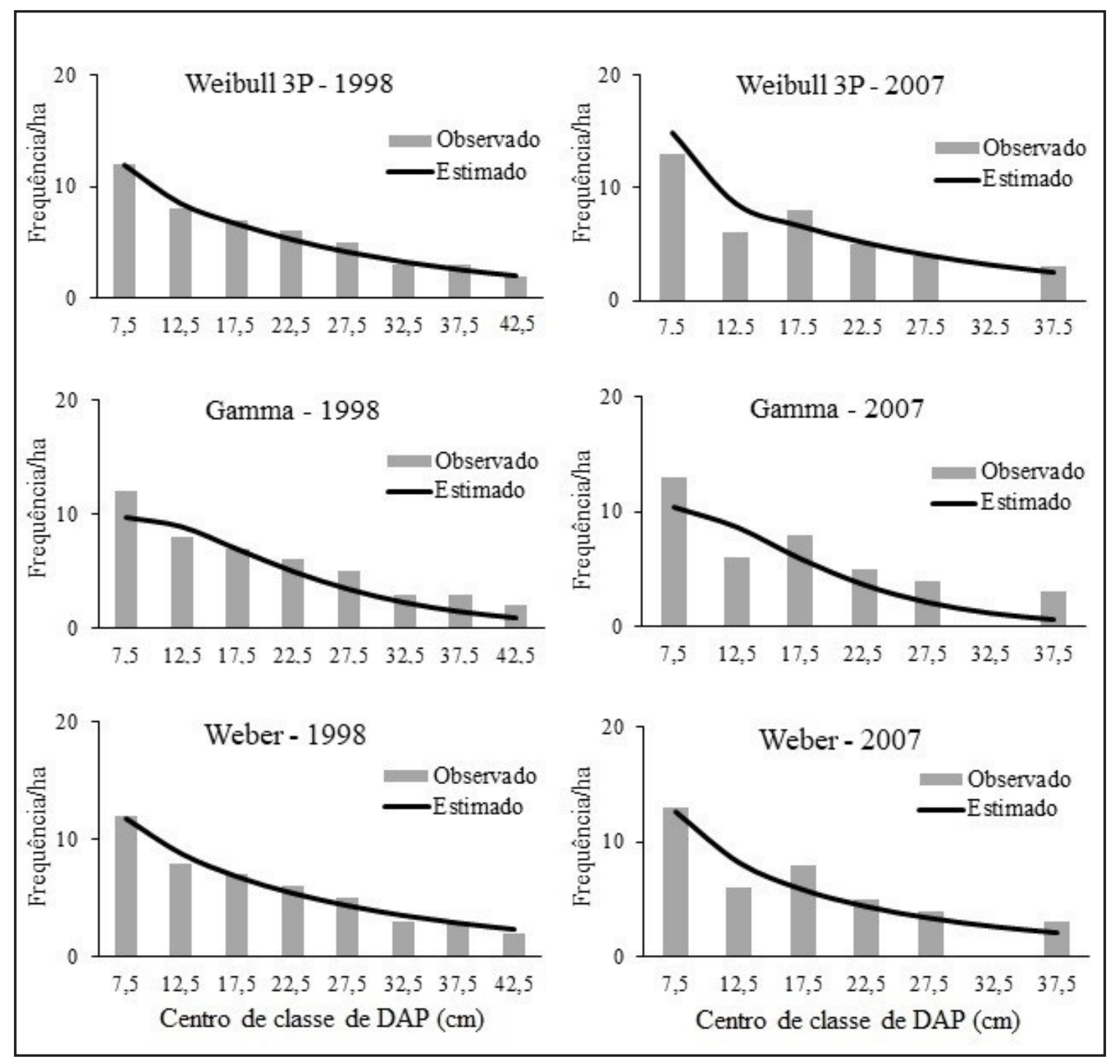

Fonte: Autores (2020) 
AnalisandoasFiguras 3e5, observa-sequeafunção de densidadedeprobabilidade Gamma foi a que apresentou menor eficiência para descrever o comportamento da distribuição diamétrica de Eschweilera coriacea e Lecythis idatimon, sendo que para ambas as espécies, essa função subestimou a frequência nas diferentes classes de diâmetro nos dois anos avaliados, principalmente, para indivíduos pertencentes às maiores classes diamétricas.

Figura 5 - Curvas de distribuição diamétrica de Lecythis idatimon estimadas pelas três funções de densidade de probabilidade, sobre o histograma de frequências observadas, um e dez anos após a exploração florestal, na Amazônia oriental, Moju-PA

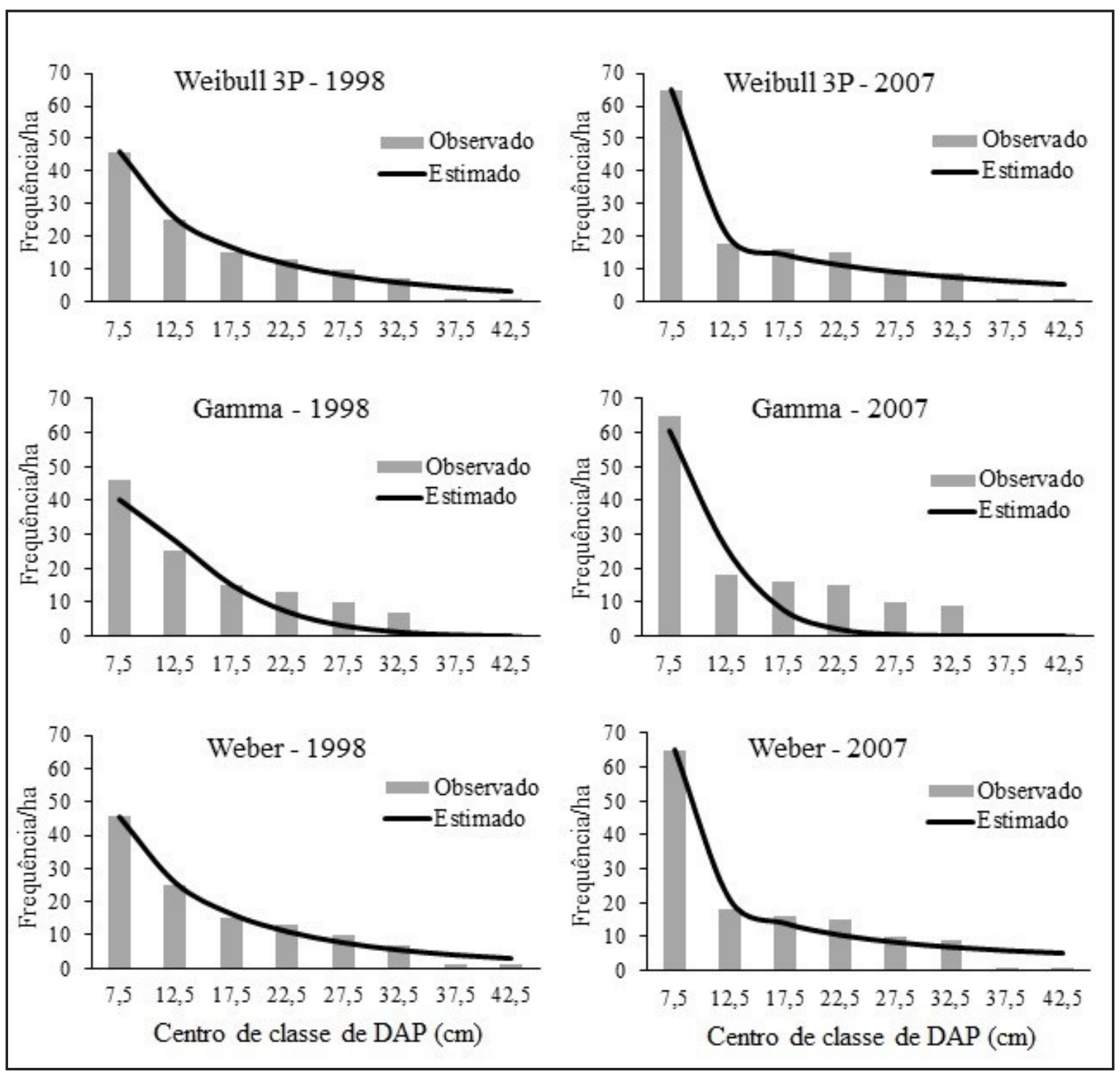

Fonte: Autores (2020) 
Analisando a Figura 4, apesar de todas as funções ajustadas apresentarem aderência confirmada no teste estatístico para Eschweilera ovata, apenas a de Weber foi satisfatória para a espécie, considerando que Weibull 3P superestimou os valores observados do primeiro centro de classe diamétrica no ano de 2007, enquanto que a função Gamma subestimou esses mesmos valores nos dois anos analisados.

Em geral, considerando o teste de Kolmogorov-Smirnov e análise gráfica, Weibull 3P foi o modelo que apresentou melhor eficiência para representar a distribuição diamétrica das espécies estudadas, um e dez anos após a colheita de madeira. Esse fato corrobora com Bailey e Dell (1973), Umaña e Alencar (1998), demonstrando que a Função Weibull 3P é altamente flexível, podendo ajustar diferentes formas e curvas tanto para florestas nativas quanto plantadas.

Vale ressaltar que a função de Weber também apresentou bons ajustes para essas espécies em ambos os anos analisados, assim como encontrado por Dalla Lana et al. (2013), ao analisarem indivíduos de Eschweilera ovata em estudo anteriormente mencionado. Weber (2006), em sua pesquisa afirmou que a principal característica da função desenvolvida consistia na sua flexibilidade para representar uma grande quantidade de indivíduos contidos na primeira classe, seguido de um decréscimo abrupto nas classes diamétricas subsequentes. Isso explica o fato dessa função ter obtido bom desempenho para as três espécies em questão, que apresentaram distribuição diamétrica semelhantes às condições que o autor descreveu.

Entretanto, apesar da função haver demonstrado melhor desempenho para descrever a distribuição de Eschweilera ovata, essa espécie não apresentou um decréscimo abrupto de indivíduos entre as classes. Tal fato também foi verificado por Machado et al. (2010), que modelando a distribuição diamétrica de quatro espécies de Lauraceae em um fragmento de Floresta Ombrófila Mista, verificaram que apesar das espécies estudadas não apresentarem decréscimo abrupto em classes diamétricas subsequentes à menor classe, a função Weber demonstrou o melhor desempenho entre os modelos ajustados, indicando uma boa flexibilidade para descrever distribuições 
de formas diversas. Além da função de Weber, esses autores constataram que as funções de SB de Johnson e Weibull 3P propiciaram os melhores desempenhos para as espécies estudadas.

Estudos sobre ajuste de funções de densidade probabilística aplicado para espécies da floresta Amazônica são raros, fato que pode ser justificado devido à grande riqueza de espécies existentes nessa região, contudo alguns dos poucos estudos que avaliam individualmente a distribuição diamétrica constataram que a função Weibull 3P é a que melhor descreve a distribuição diamétrica de determinadas populações de espécies da região (CYSNEIROS et al., 2014; RIBEIRO et al., 2014; SANTOS et al., 2013).

Nascimento et al. (2012) afirmam que a função Weibull possui características matemáticas que o fazem ser um ferramental descritivo muito flexível, podendo assumir, conforme os valores de seus coeficientes, várias formas e assimetrias, o que possibilita seu emprego na descrição de diferentes relações dendrométricas ou tipologias florestais. Todavia, Higuchi et al. (2012) constataram que não foi encontrado nenhum registro de que a função de Weibull é utilizada no setor produtivo, apesar de sua eficácia ter sido comprovada por diversos autores em diferentes populações florestais.

\section{CONCLUSÃO}

As curvas de distribuição diamétrica se apresentaram na forma exponencial negativa ("J invertido"), um ano após a colheita florestal para as três espécies estudadas. Contudo, dez anos após a exploração, as espécies não mantiveram esse comportamento, apresentando distribuição decrescente, porém de forma menos acentuada, com exceção de Eschweilera coriaceae que manteve sua distribuição diamétrica similar nos dois períodos analisados.

Dentre os modelos testados, considerando os dois anos de avaliação, Weibull 3P apresentou as melhores estatísticas de ajuste e maior precisão para descrever a distribuição diamétrica de Eschweilera coriacea e Lecythis idatimon. Weber também 
se mostrou aderente, demonstrando maior eficiência para descrever a distribuição diamétrica de Eschweilera ovata. A função Gamma foi considerada a função de densidade probabilística menos eficiente para descrever a distribuição diamétrica das espécies nos períodos avaliados.

\section{REFERÊNCIAS}

AISN Software. Table Curve 2D. Jandel Scientific, 1994.

ARAÚJO, C. B. Composição florística e estrutura de uma floresta densa de terra firme explorada seletivamente no município de Moju, Pará. 2011. 123 f. Dissertação (Mestrado em ciências florestais) - Universidade Federal Rural da Amazônia, Belém, 2011.

ASSMANN, E.The principles of forest yield: studies in the organic production, structure, increment and yield of forest stands. Braunschweig: Pergamon Press, 1970. 506 p.

BAILEY, R. L.; DELL, T. R. Quantifying diameter distributions with the Weibull function. Forest Science, Bethesda, v. 19, n. 2, p. 97-104, 1973.

COSTA, D. H. M. et al. Potencial madeireiro de floresta densa no município de Mojú, Estado do Pará. Belém: Embrapa-CPATU, 1998. 33 p.

CYSNEIROS, V. C. et al. Distribuição diamétrica de espécies da Floresta Ombrófila Densa no Sul do Estado do Rio de Janeiro. Pesquisa Florestal Brasileira, Colombo, v. 37, n. 89, p. 1-10, 2017.

DALLA LANA, M. et al. Distribuição Diamétrica de Eschweilera ovata em um Fragmento de Floresta Ombrófila Densa - Igarassu, PE. Floresta, Curitiba, v. 43, n. 1, p. 59-68. 2013.

HARPER, J. L. Population biology of plants. London: Academic Press, 1990. 892 p.

HIGUCHI, F. G. et al. Influência do tamanho da parcela na precisão da função de distribuição diamétrica de Weibull na floresta primária da Amazônia Central. Floresta, Curitiba, v. 42, n. 3, p. 599-606, 2012.

INSTITUTO BRASILEIRO DE GEOGRAFIA E ESTATÍSTICA. Manual técnico da vegetação brasileira. Rio de Janeiro: Instituto Brasileiro de Geografia e Estatística, 2012. 271 p.

JARDIM, F. C. S.; VASCONCELOS, L. M. R. Dinâmica da regeneração natural de Rinorea guianensis Aublet, em uma floresta tropical primária explorada seletivamente, Moju (PA). Revista de Ciências Agrárias, Belém, v. 45, p. 121-134, 2006.

LIMA, P. L. et al. Q. Regeneration of five commercially-valuable tree species after experimental logging in an Amazonian forest. Revista Árvore, Viçosa, v. 26, n. 5, p. 567-571, 2002.

MACHADO, S. A. et al. Modelagem da distribuição diamétrica de quatro espécies de Lauraceae em um Fragmento de Floresta Ombrófila Mista. Revista Ciências Exatas e Naturais, Guarapuava, v. 12 n. 1, p. 91-105, 2010. 
MACHADO, S. A. et al.Funções de distribuição diamétrica em um fragmento de Floresta Ombrófila Mista. Ciência Rural, Santa Maria, v. 39, n. 8, p. 2428-2434, 2009.

MARQUARDT, D. W. An algorithm for least squares estimation of nonlinear parameters. Journal of the Society for Industrial and Applied Mathematics, Auckland, v. 11, n. 2, p. 431-441, 1963.

MORI, S. A.; PRANCE, G. T. Lecythidaceae - Part II: The Zigomorphic-flowered New World Genera (Couroupita, Corythophora, Bertholletia, Couratari, Eschweilera \& Lecythis). Flora Neotropica: Monograph 21 (II). New York Botanical Garden, New York, 1990. 376 p.

MORI, S. A. A família da castanha-do-Pará: símbolo do Rio Negro. In: OLIVEIRA, A. A.; DALY, D. (Eds.). Florestas do Rio Negro. São Paulo e New York: Companhia das Letras, Universidade Paulista e The New York Botanical Garden, p. 119-141, 2001.

NASCIMENTO, R. G. M. et al. Modelo de projeção por classe diamétrica para florestas nativas: enfoque na função probabilística de Weibull. Pesquisa Florestal Brasileira, Colombo, v. 32, n. 70, p. 209-219, 2012.

ORELLANA, E. et al. Modelagem da distribuição diamétrica de espécies florestais em um fragmento de floresta ombrófila mista. Revista Árvore, Viçosa, v. 38, n. 2, p. 297-308, 2014.

REIS, L. P. Efeito da exploração de impacto reduzido na dinâmica da densidade, dominância e crescimento de espécies de Sapotaceae em floresta de terra firme no município de Moju, estado do Pará. 2012. Dissertação (Mestrado em Ciências Florestais) - Universidade Federal Rural da Amazônia, Belém, 2012.

RIBEIRO, A. et al. Estrutura da distribuição diamétrica em plantio experimental de candeia (Eremanthus erythropappus (DC.) MacLeish). Ciência Florestal, Santa Maria, v. 24, n. 4, p. 10551065, 2014.

SANTOS, E. S. et al. Distribuição Diamétrica para Virola surinamensis (Rol.) na Floresta Estadual do Amapá-FLOTA/AP. Revista de Biologia e Ciências da Terra, São Cristóvão, v. 13, n. 1 p. 34-47, 2013.

SCOLFORO, J. R. S. Biometria florestal: modelos de crescimento e produção florestal. Lavras, UFLA/FAEPE, 2006. 393 p.

SECRETARIA DE ESTADO DE MEIO AMBIENTE E SUSTENTABILIDADE. Extração e Comércio de Toras de Madeira Nativa por Essência. Disponível em: https://monitoramento.semas. pa.gov.br/sisflora/relatorios.html. Acesso em 28 de abril de 2018.

SMITH, N. P. et al. Lecythis ibiriba (Lecythidaceae), a new combination from northeastern Brazil. Journal of the Torrey Botanical Society, New York, v. 139, n. 4, p. 447-451, 2012.

SMITH, N. P. et al. Conservation assessment of Lecythidaceae from eastern Brazil. Kew bulletin, [s.I], v. 71, n. 1, p. 14, 2016.

TÉO, S. J. et al. Modelagem da distribuição diamétrica de um fragmento de Floresta Ombrófila Mista em Lebon Régis, SC. Floresta, Curitiba, v. 45, n. 2, p. 337-348, 2015. 
TER STEEGE, H. et al. Continental scale patterns of canopy tree composition and function across Amazônia. Nature, London, v. 443, p. 444- 447, 2006.

TER STEEGE, H. et al. Hyperdominance in the Amazonian tree flora. Science, New York, v. 342, n. 6156, p. 1243092, 2013.

UMAÑA, C. L. A.; ALENCAR, J. C. Distribuições diamétricas da floresta tropical úmida em uma área no município de Itacoatiara-AM. Acta amazonica, v. 28, n. 2, p. 167-190, 1998.

VERÍSSIMO, A.; PEREIRA, D. Produção na Amazônia Florestal: características, desafios e oportunidades. Parcerias Estratégicas, Brasília, v. 19, n. 38, p. 13-44, 2014.

WEBER, S. H. Desenvolvimento de nova função densidade de probabilidade para avaliação de regeneração natural. 2006. 74 f. Dissertação (Mestrado em Engenharia Florestal) Universidade Federal do Paraná, Curitiba, 2006.

\section{Contribuição de Autoria}

\section{1 - Loarena Leal Cruz}

Engenheira Florestal, Ma.

https://orcid.org/0000-0002-3174-3021•loarenacruz@gmail.com

Contribuição: Curadoria de dados, Análise Formal, Visualização, Escrita - primeira redação

\section{2 - Nelson Yoshihiro Nakajima}

Engenheiro Florestal, Dr.

https://orcid.org/0000-0002-5998-1128•nelson.nakajima@ufpr.br

Contribuição: Curadoria de dados, Análise Formal, Visualização, Escrita - primeira redação, Escrita - revisão e edição

\section{3 - Renildo Medeiros da Silva}

Engenheiro Florestal

https://orcid.org/0000-0001-8007-9780 • renildomedeiros.florestal@gmail.com

Contribuição: Escrita - primeira redação 


\section{4 - Roberto Tuyoshi Hosokawa}

Engenheiro Florestal, Dr.

https://orcid.org/0000-0002-2595-2361 •roberto.hosokawa@pesquisador.cnpq.br

Contribuição: Escrita - primeira redação, Escrita - revisão e edição

\section{5 - Fernando Cristóvam da Silva Jardim}

Engenheiro Florestal, Dr., Professor

https://orcid.org/orcid.org/0000-0002-9855-759X•fernando.jardim@ufra.edu.br

Contribuição: Curadoria de dados, Análise Formal, Visualização, Escrita - revisão e edição

\section{6 - Ana Paula Dalla Corte}

Engenheira Florestal, Dra.

https://orcid.org/0000-0001-8529-5554•napaulacorte@gmail.com

Contribuição: Curadoria de dados, Análise Formal, Visualização, Escrita - revisão e edição

\section{Como citar este artigo}

Cruz, L. L.; Nakajima, N. Y.; Silva, R. M.; Hosokawa, R. T.; Jardim, F. C. S.; Corte, A. P. D. Distribuição diamétrica de três espécies de Lecythidaceae após exploração de impacto reduzido na Amazônia Oriental. Ciência Florestal, Santa Maria, v. 31, n. 1, p. 171-190, 2021. DOI 10.5902/1980509836011. Disponível em: https://doi.org/10.5902/1980509836011. Acesso em: xx mês abreviado 202x. 